\title{
M3 - Mathe im April, Mai und Advent
}

\author{
Stephanie Schiemann
}

In diesem Artikel geht es um die Regeln, Hintergründe und Statistiken der Mathe-Challenges für Schüler*innen, Lehrer*innen und sonstige Interessierte, die die Mathe im Leben gGmbH seit Jahren in Kooperation mit der DMV sehr erfolgreich ausrichtet. Sie erfahren auch, wie Sie sich selbst mit wenig Aufwand beteiligen können.

Rekordzahlen bei den digitalen Mathe-Challenges in der Zeit des eingeschränkten Schulbetriebs in der BMBFInitiative „Wir bleiben schlau!“. Im Advent waren es $40 \%$ Teilnehmende mehr als sonst, 184580 .

Jeder kann beim täglichen Knobeln im Advent mitmachen, jeder kann Ideen für die Aufgaben liefern und somit über 170 ooo Kindern und Jugendlichen und 7000 Lehrkräften angewandte Mathematik näher bringen.

\section{Die Mathe-Challenges}

2020 gab es ausnahmsweise drei digitale Mathe-

Challenges für alle Schüler*innen und Lehrkräfte.

In der Zeit der Schulschließungen und des eingeschränkten Schulbetriebs haben wir kurzentschlossen in der BMBF-Initiative „Wir bleiben schlau! - Die Allianz für MINT-Bildung zu Hause“ die beiden Extra-MatheChallenges „Mathe im April“ und „Mathe im Mai“ auf die Beine gestellt. Sie liefen nach dem Vorbild von „Mathe im Advent". Alle, Schüler*innen wie Erwachsene, hatten die Möglichkeit, alleine teilzunehmen. Lehrkräfte konnten im April und Mai auch kostenlos ihre Klassen anmelden und im Klassenverbund täglich Mathe-Aufgaben von zu Hause lösen. Über 1 oo ooo Schüler*innen nutzten diese Chance! Für die zwei Besten stiftete die DMV im Mai ein iPad.

„Mathe im Advent" (kurz: MiA) ist kein Talentwettbewerb, sondern ein Angebot für alle, die spielerisch Mathematik entdecken möchten. Im Gegensatz zum RoutinenTraining im normalen Schulunterricht fördert MiA viele wichtige mathematische Kompetenzen wie kreatives Problemlösen, Mustererkennen, Verallgemeinern, Schätzen und logisches Denken. Mit den Aufgaben und umfangreichen Lösungen zeigen wir Kindern, Jugendlichen, Eltern und Großeltern, dass Mathematik tatsächlich Spaß machen kann und sinnvoll in vielen Bereichen des Lebens angewendet wird. Auch diejenigen, die Mathe nicht mögen oder nur noch wenig erinnern, haben viel Spaß an unseren „Mathe im Advent"-Aufgaben.

\section{Wie läuft's?}

MiA ist ein Online-Wettbewerb, der sich in der Adventszeit vom 1. bis zum 24. Dezember an alle Schüler und Schülerinnen der Klassenstufen 2-10 wendet. Auf www.mathe-im-advent.de werden spannende, anwendungsorientierte Mathe-Wichtel-Aufgaben in zwei Niveaustufen für die Klassenstufen 4-6 und 7-9 angeboten.

Mitmachen können übrigens neben den Schüler*innen und Lehrkräfte auch alle sonstigen Interessierten. Melden Sie sich dazu einfach als „Spaßspieler“ an. Sie können dann die Aufgaben beider Niveaustufen lösen und erhalten am Ende auch eine Urkunde. Jährlich knobeln oftmals ganze Familien, Eltern und Großeltern mit.

„Mathe im Advent" wird von der Mathe im Leben gemeinnützigen GmbH in Kooperation mit der DMV ausgerichtet. Premiumpartner ist die Gisela und Erwin Sick Stiftung, Hauptförderer die Klaus Tschira Stiftung und weitere Förderer sind Berliner helfen e. V. und die Stiftung Standortsicherung Lippe. Parallel dazu gibt es für die Oberstufenschüler*innen und Erwachsenen den digitalen Mathekalender vom Berliner Forschungszentrum MATH+.

\section{Die "Mathe-im-Advent"-Aufgabengeschichten}

Die täglich wechselnden Geschichten spielen im fiktiven Weihnachtswichteldorf. Es macht Spaß, die Texte rund um die Wichtel, Rentiere und den Weihnachtsmann zu lesen und die liebevollen Comic-Illustrationen anzuschauen. Quasi nebenbei löst man mathematische Probleme und übt Textverständnis. Jeden Tag lernen die Teilnehmenden die Charaktere besser kennen und tauchen tiefer in die Wichtelwelt ein - ähnlich wie bei Harry Potter oder Seifenopern. Die fiktive Szenerie ist dabei immer so gestaltet, dass ein Kind die Handlungen und daraus resultierenden mathematischen Fragestellungen als sinnvoll empfinden kann. Überträgt das Kind die Geschichten auf sein eigenes Leben, kann es nachvollziehen, wo die Mathematik nützlich ist und überall verwendet wird. Darüber hinaus werden gleichberechtigte Rollenvorbilder für Mädchen und Schüler*innen mit Migrationshintergrund vermittelt, die Führungspositionen innehaben, rechnen oder programmieren können und wichtige Jobs zur Rettung der Umwelt oder zum Schutz der Tiere ausführen.

\section{Wettbewerbskonzept}

Wir glauben, dass eine nachhaltige Entwicklung von Motivation und Kompetenzen in der Mathematik nicht in einem eintägigen, klausur-artigen Projekt erreicht werden kann. Deshalb ist MiA als Spiel konzipiert, in dem Kinder und Jugendlichen 24 Tage lang spannende mathematische Inhalte erforschen und entdecken können. Um verschiedene Zugänge zu ermöglichen, haben wir mehrere Arten der Teilnahme entwickelt, mit und ohne Gewinnspiel, alleine oder als Gruppe:

Einzelspiel: Nach der Registrierung eines Accounts können die Schüler*innen entweder nur aus Spaß teilnehmen (nutzt ca. 1/3) oder im Einzelspiel gegen alle anderen Kinder spielen und spannende Preise gewinnen. 
Klassenspiel: Lehrerinnen können ihre Klassen zudem virtuell in unserem System anlegen und ihre Schüler*innen zur gemeinsamen Teilnahme im Klassenspiel einladen. Dies ist das Herz des Projekts, denn damit rückt die Mathematik im gesamten Dezember ins Zentrum des Klassen- und Schullebens.

MiA fördert zusätzlich zu den mathematischen Kompetenzen das Teamwork sowie präzises, folgerichtiges Argumentieren und das Durchhaltevermögen. In diesem Format spielen auch Kinder mit, die sonst nie an einem Mathe-Wettbewerb teilnehmen würden. Schüler*innen im Klassenspiel bleiben zudem durchschnittlich länger dabei und lösen mehr Aufgaben richtig als Einzelspieler*innen. Auch Lehrer*innen gewinnen neue Motivation und entdecken mathematische Inhalte und Anwendungen, die ihren zukünftigen Unterricht bereichern können. Die besten Klassen und Schulen gewinnen Preise oder Pokale.

\section{Bundesministerin hat die Schirmherrschaft}

Die Mathe-Adventskalender von Mathe im Leben und MATH+ stehen unter der Schirmherrschaft der Bundesministerin für Bildung und Forschung, Anja Karliczek. In dem Teilnehmerrekord 2020 sieht sie eine positive Entwicklung für die MINT-Fächer:

Deutschland ist Innovationsland und auf die guten und kreativen Ideen der Menschen in unserem Land angewiesen. Junge Menschen, die sich für Mathematik und Naturwissenschaften begeistern können, sind deshalb der Garant dafür, dass wir unseren Lebensstandard auch in Zukunft halten können. Sie erfinden und entwickeln Dinge, von denen alle Menschen profitieren. Die große Resonanz freut mich deshalb sehr. Der Anstieg der Teilnehmerzahl um 4o Prozent lässt hoffen, dass das Interesse an der Mathematik und den Naturwissenschaften in den kommenden Jahren weiter anwächst. Die digitalen Mathe-Adventskalender sind ein attraktives Angebot, das Kinder und Jugendliche altersgerecht und mit viel Spaß an die MINTFächer und an das sinnvolle Arbeiten mit digitalen Medien heranführt.
Historie

Die Idee des digitalen Mathekalenders für die Grundund Mittelstufen ist im Jahr der Mathematik 2008 entstanden, als Günter M. Ziegler DMV-Präsident war. Das Wissenschaftskommunikationsprojekt hat inzwischen 13-mal stattgefunden. Von 2010-2015 wurde „Mathe im Advent" vom DMV-Netzwerkbüro Schule-Hochschule koordiniert. Seit 2016 hat die eigens dafür gegründete gemeinnützige $\mathrm{GmbH}$ Mathe im Leben die Organisation übernommen.

Kinder sollen möglichst früh positive Erfahrung mit Mathematik machen, erkennen, dass Mathe Spaß machen kann. Deshalb können bei MiA „Frühstarter“ ab der 2. Klasse teilnehmen. In den Lösungen bieten wir zur Einbettung des Themas oft einen „Blick über den Tellerrand" oder eine „Mathematische Exkursion“. Uns ist wichtig zu vermitteln, dass Mathematik nicht nur Rechnen ist und viel mehr dazu gehört als in der Schule gelernt wird.

\section{Aufgabenwettbewerb}

Gemeinsam mit der gesamten Mathematik-Community sammelt das Team für die nächste Runde schon jetzt Aufgabenideen und ruft jährlich den "Aufgabenwettbewerb“ aus. Mitmachen können alle: Wissenschaftler*innen, Lehrkräfte, Eltern wie Schüler*innen. Einsendeschluss ist der 30. Juni 2021. Jede und jeder kann so mithelfen, mit aktuellen mathematischen Themenfeldern das Bild der Mathematik für alle Teilnehmenden zu erweitern und der Öffentlichkeit ein interessantes Mathematisches Einsatzgebiet nahe zu bringen. Das Thema auf Schulniveau herunterzubrechen, ist eine Herausforderung, die sich lohnt, denn täglich lesen über 500 ooo Personen die Aufgaben. In einer Spielrunde haben wir weit über 30 Millionen Seitenaufrufe. In 12/2020 waren wir mit MiA beim SimliarWebRank in der Category: Science (weltweit) \#50.

Reichen Sie uns bitte per E-Mail an info@mathe-imadvent.de Ihre Aufgabenidee mit einer Fragestellung, die sich mit Multiple-Choice beantworten lässt, sowie einer Lösungsskizze ein. Wir freuen uns auch über Ideen zu einer „Mathematischen Exkursion“ bzw. einem „Blick über

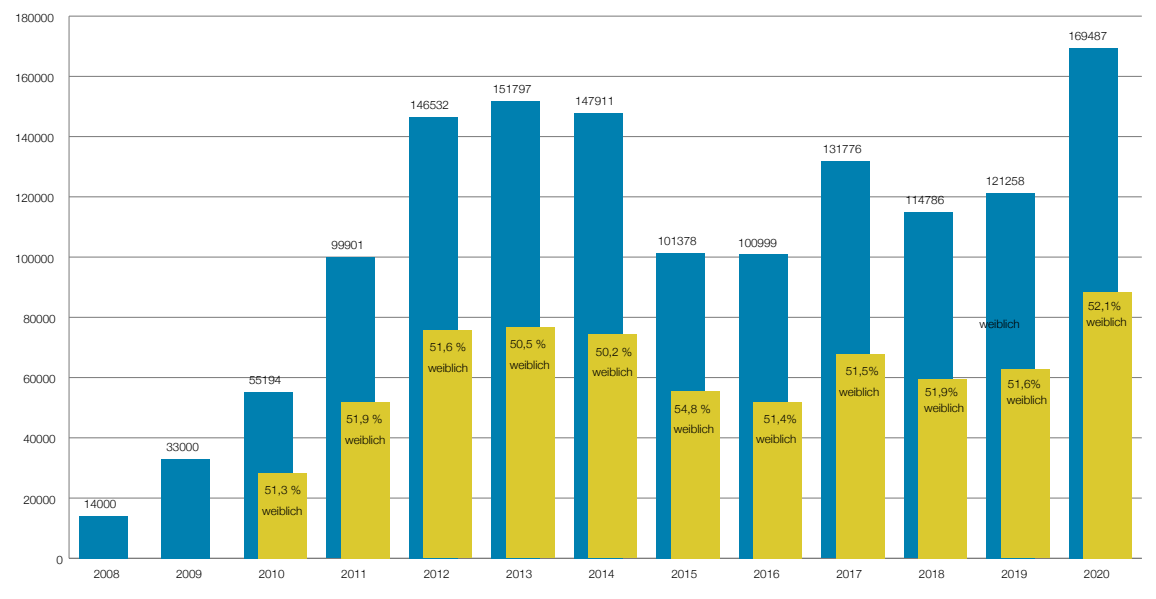

Teilnehmerzahlen „Mathe im Advent“ (alle Nutzergruppen). Seit 2008 gab es insgesamt 1500 360 Teilnehmer*innen. 


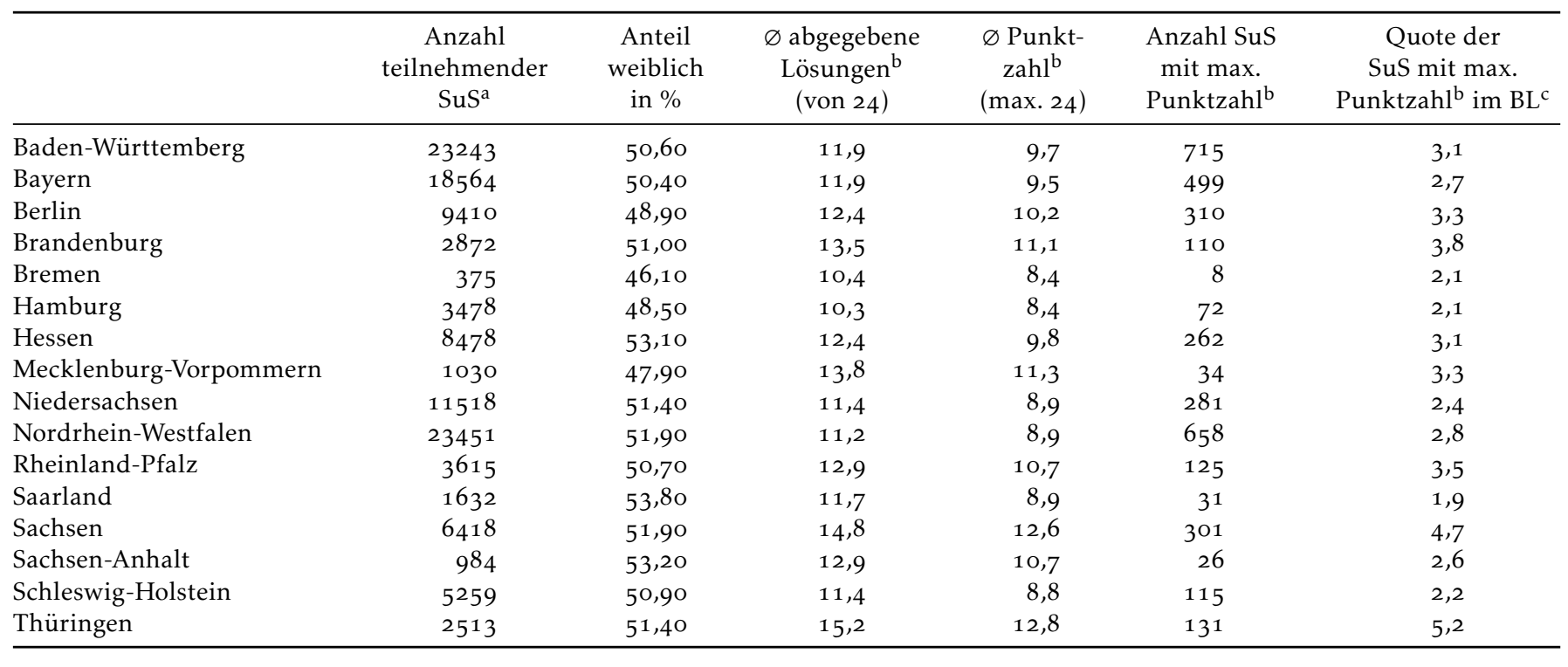

a. SuS: Schülerinnen und Schüler

b. jeweils ohne Jokereinsatz

c. Bundesland

den Tellerrand“. Zahlreiche Beispielaufgaben finden Sie im Web oder in den Büchern "Die Mathe-Wichtel“.

Die „Mathe im Advent“-Jury wird die schönsten, zum Format passenden Aufgaben für den Adventskalender 2021 auswählen. Die Bewertung aller Teilnehmenden bestimmt dann im Dezember die beliebtesten Aufgaben beider Kalender, deren Autor*innen dann - sofern es Corona zulässt - auf der „Mathekalender“-Preisverleihung geehrt werden. Siegerin im Aufgabenwettbewerb 2020 (Kalender 7-9) war die Bochumer Lehrerin Dr. Gloria
Becker. Sie erhielt den neuen Wanderpokal. Zudem ehrte die DMV sie als Mathemacherin der Monate Januar/ Februar.

„Mathe im Advent": Statistik

Seit 2008 haben bereits mehr als 1,5 Millionen Schülerinnen und Schüler an „Mathe im Advent" teilgenommen, davon waren jeweils mehr als $50 \%$ weiblich. Wie man im Diagramm auf auf der vorherigen Seite sieht, gab es 2020 mit 170000 einen absoluten Teilnahmerekord.

Schul-, Klassen- und Lehrerstatistik „Mathe im Advent 2020“ nach Bundesländern

\begin{tabular}{|c|c|c|c|c|c|c|}
\hline & $\begin{array}{l}\text { Gesamt- } \\
\text { zahl } \\
\text { aller } \\
\text { Schulen }^{\text {a }}\end{array}$ & $\begin{array}{c}\text { Anzahl } \\
\text { teilnehmender } \\
\text { Schulen } \\
\text { mit mindestens } \\
\text { einem } / \mathrm{r} \text { SuS }\end{array}$ & $\begin{array}{l}\text { Anzahl } \\
\text { teilnehmender } \\
\text { Schulen } \\
\text { mit mindestens } \\
\text { einer Klasse }\end{array}$ & $\begin{array}{c}\text { Anteil } \\
\text { teilnehmender } \\
\text { Schulen } \\
\text { am Klassenspiel } \\
\text { in \% }\end{array}$ & $\begin{array}{c}\text { Anzahl } \\
\text { teilnehmen- } \\
\text { der Lehrer }\end{array}$ & $\begin{array}{c}\text { Anteil der } \\
\text { weiblichen } \\
\text { Lehrkräfte } \\
\text { in \% }\end{array}$ \\
\hline Baden-Württemberg & 4708 & 1013 & $45^{\circ}$ & 9,6 & 1222 & 63,9 \\
\hline Bayern & 4633 & 1092 & 260 & 5,6 & 989 & 63,9 \\
\hline Bremen & 187 & 53 & 9 & 4,8 & 30 & 66,7 \\
\hline Hamburg & 424 & 144 & 42 & 9,9 & 163 & 61,3 \\
\hline Hessen & 2464 & 528 & 129 & 5,2 & 403 & 66,5 \\
\hline Mecklenburg-Vorpommern & 710 & 133 & 19 & 2,7 & 62 & 66,1 \\
\hline Niedersachsen & 3245 & 602 & 164 & 5,1 & 537 & 59,4 \\
\hline Nordrhein-Westfalen & 5019 & 1449 & 354 & 7,1 & 1141 & 63,7 \\
\hline Schleswig-Holstein & 1236 & 246 & 76 & 6,1 & 256 & 57,0 \\
\hline Thüringen & 881 & 208 & 36 & 4,1 & 107 & 57,9 \\
\hline
\end{tabular}

a. Grund-, Haupt-, Real-, Gesamt-, Gemeinschafts-, Sekundar-, Waldorf- und Förderschulen sowie Gymnasien öffentlicher und privater Träger 


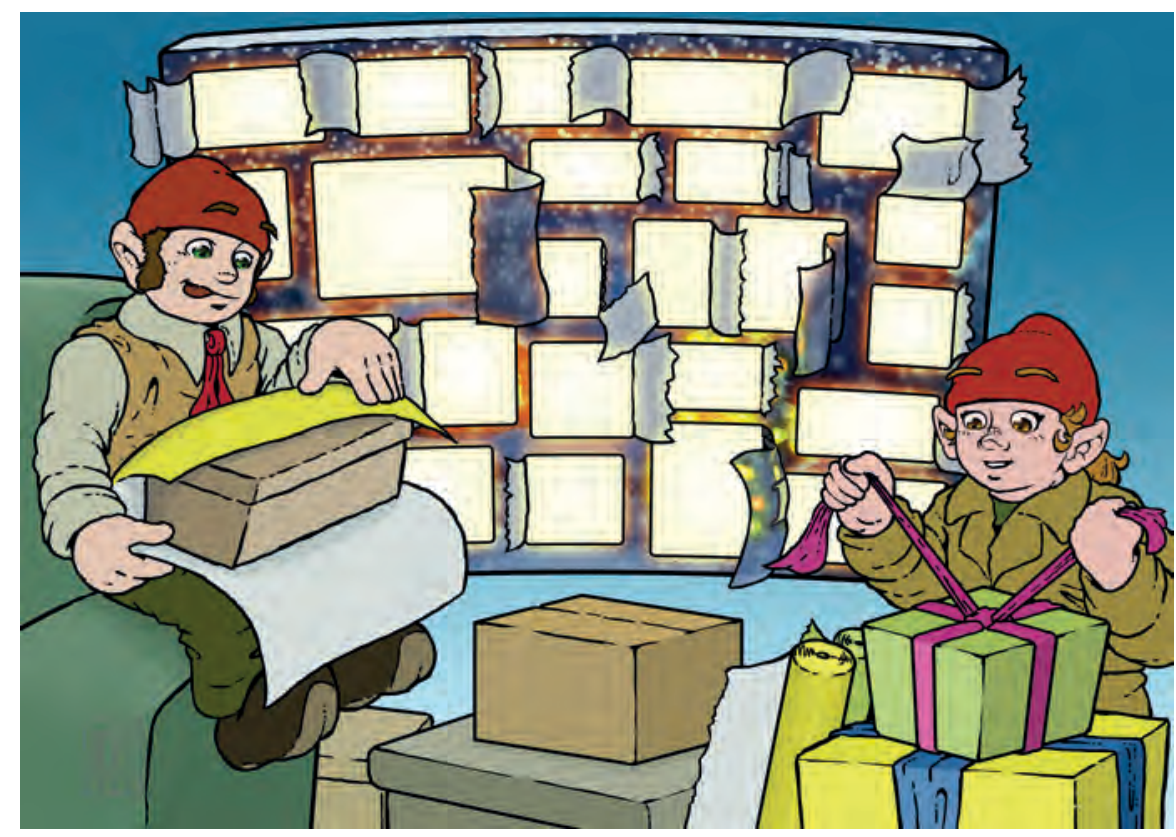

Die Mathe-Wichtel verpacken Geschenke für alle „Mathe im Advent“-Gewinner*innen

Die Teilnahme allein - im sogenannten Einzelspiel - ist schon immer kostenfrei. Das Klassenspiel - in der Gruppe mit einer betreuenden Lehrkraft - war bis zum Jahr 2014 ebenfalls kostenfrei und wurde dann nach dem Ende der BMBF-Förderung auf Teilnahmegebühren umgestellt. Deshalb haben wir 2015 etwa 50 ooo Schüler ${ }^{*}$ innen und 5000 Lehrkräfte im Klassenspiel verloren. Seit dem Jahr 2015 können wir das Klassenspiel nur noch in den geförderten Regionalprojekten (derzeit im Regierungsbezirk Freiburg - PLZ 77X, 78x und 79x, Berlin-Neukölln und im Märkischen Viertel sowie im Kreis Lippe) kostenfrei anbieten. Wir versuchen, die regionalen Förderungen auszuweiten, um zukünftig wieder mehr Schulen das kostenfreie Klassenspiel anzubieten. Für Anregungen diesbezüglich sind wir sehr dankbar.

Auf der Deutschlandkarte auf Seite 184 sehen Sie die Verteilung der teilnehmenden Schüler*innen bei den Ka- lendern 4-6 und 7-9. Nun stellen Sie sich vielleicht die Frage, wie viele Schulen, Klassen, Lehrer*innen und Schüler*innen mitmachen. Wie lange halten sie durch und wie viele lösen tatsächlich alle 24 Aufgaben korrekt? Dazu gehört ein enormes Durchhaltevermögen, was dieses Mal durch den vorzeitigen Lockdown etliche Tage nicht mehr von der Lehrkraft begleitet werden konnte. Einen kleinen Einblick bieten die beiden Bundeslandstatistiken auf der vorangehenden Seite. Wer sich für mehr Daten interessiert, z. B. aus unserer Vor- und Nachherumfrage tausender Schüler ${ }^{\star}$ innen, Lehrkräfte und Spaßspieler, möge mich ansprechen. Wir suchen fortwährend sowohl Studierende als auch Wissenschaftler*innen, die Interesse haben mitzuarbeiten, z. B. bei der fachlichen Überprüfung der fertigen Aufgaben oder im wissenschaftlichen Beirat von "Mathe im Advent", oder die Forschungsarbeiten über unser Projekt schreiben (lassen) möchten.

Stephanie Schiemann,

Mathe im Leben $g \mathrm{GmbH}$, c/o Freie Universität Berlin, Institut für Mathematik, Arnimallee 2, 14195 Berlin schiemann@mathe-im-leben.de

Stephanie Schiemann, Studienrätin, 20 Jahre Mathematik- und Sport-Lehrerin an verschiedenen Schulformen in Niedersachsen, ehem. Schulbuchautorin, Leiterin der Talentförderung Mathematik und zahlreicher Lehrerfortbildungen, neun Jahre Leiterin des $D M V$-Netzwerkbüros Schule-Hochschule und jetzt geschäftsführende Gesellschafterin der Mathe im Leben $g G m b H$. 
Am 25. Dezember findet jedes Jahr der große Weihnachtsball des Wichteldorfs statt. Die Wichtel Orlandie und Albert bereiten das Fest vor. Bei der Besichtigung des großen Ballsaals haben die beiden eine wichtige Entdeckung gemacht: Der Parkettboden ist an vielen Stellen kaputt. Da können die Wichtel beim Tanzen stolpern. Die beiden haben deshalb Wichtel Berenike um Hilfe gebeten. Die Tischlerin soll ein schönes neues Parkett auslegen.

Als Berenike heute im Ballsaal ankommt, sind die alten Bodenbretter schon fast alle entfernt. Für den neuen Boden hat sie zwei verschiedene Rautenformen dabei, die sie als Parkettstücke verwenden möchte - breitere Rauten und schmalere Rauten (siehe 1 . Bild). Sie unterscheiden sich in den Winkeln, doch die Seiten sind alle gleich lang. Die Winkel sind so gewählt, dass sie als Parkett ohne Lücken aneinandergelegt werden können.

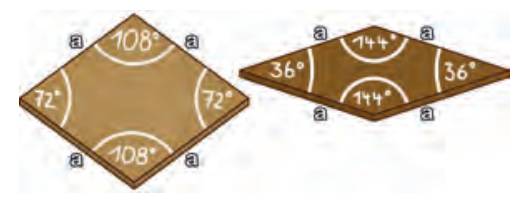

Berenike möchte ein schönes Parkettmuster mit den beiden Rauten erzeugen. Dafür beginnt sie im Mittelpunkt des Raums mit fünf breiten Rauten, die genau aneinanderpassen, denn $5 \cdot 72^{\circ}=360^{\circ}$ (siehe Bild). Um sie herum legt sie die schmalen Rauten im Kreis. Sie hat jetzt einen ,inneren Kreis“ aus fünf breiten und fünf schmalen Rauten.

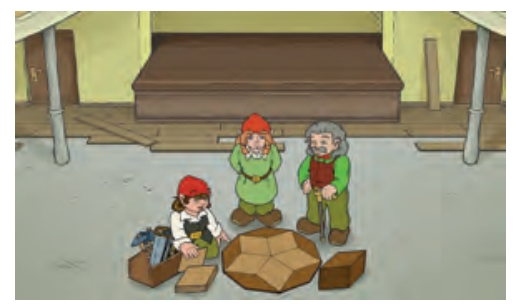

Um den „inneren Kreis“ möchte sie weitere Ringe legen, immer einen nach dem anderen außen drumherum. So soll sich das Muster bis zum Rand des Raumes ausbreiten. Doch Berenike, Albert und Orlandie sind unterschiedlicher Meinung, wie sie mit den beiden Rautenformen den nächsten Ring legen können.
Berenike meint: „Das geht mit beiden Rautenformen, wenn wir sie immer abwechselnd legen." Albert ist sich sicher: „Nein, ich glaube das geht nur mit den breiteren Rauten." Orlandie antwortet: „Ich nehme an, der nächste Ring kann nur mit den schmalen Rauten gelegt werden. Die sind eh viel schöner." Doch wer hat recht?

Mit welchen Rautenformen kann der nächste Ring gelegt werden, so dass keine Lücken entstehen? Nur eine Aussage ist richtig.

Hinweis. Der neue Ring muss vollständig sein und muss den „inneren Kreis“ komplett umschließen. Zwei benachbarte Rauten des neuen Rings müssen also immer eine gemeinsame Kante haben. Wie der äußere Rand dann aussieht, spielt keine Rolle. Probiere es selbst aus, indem du die beiden Rauten konstruierst..

a. Der nächste Ring lässt sich nur legen, wenn man ausschließlich die breiten Rauten benutzt.

b. Der nächste Ring lässt sich nur legen, wenn man ausschließlich die schmalen Rauten benutzt

c. Der nächste Ring lässt sich nur legen, wenn man die beiden Rautenformen immer im Wechsel auslegt.

d. Der nächste Ring lässt sich mit allen drei Möglichkeiten legen. Das heißt: Es funktioniert, wenn man ausschließlich breite Rauten benutzt, wenn man ausschließlich schmale Rauten benutzt und auch wenn man beide Rauten immer abwechselnd legt.

\section{Blick über den Tellerrand}

Penrose-Parkett vom Nobelpreisträger Penrose

Solche Rautenparkette werden nach seinem Erfinder Roger Penrose als Penrose-Parkett bezeichnet. Penrose ist ein britischer Mathematiker und Physiker (Jahrgang 1931). Er hat im Jahr 2020 für eine Entdeckung, zum Thema Schwarze Löcher den Nobelpreis für Physik bekommen.

Penrose entdeckte 1973 die aperiodischen Kachelmuster, mit denen du in dieser Aufgabe selbst Entdeckungen machen konntest. Mit den beiden
Rauten lässt sich auf verschiedenen Arten eine Fläche lückenlos parkettieren, du kannst in einem Raum viele verschiedene Parkettmuster mit den beiden Rauten verlegen. Das Grundschema ist periodisch, wiederholt sich also nach einer gewissen Zeit immer wieder. Stellst du dir einen unendlich großen Raum vor - also eine unendlich große Fläche - könntest du diese mit einem immer wiederholenden PenroseMuster auslegen.

Es gibt mehrere Varianten dieser Penrose-Kacheln. Die bekannteste ist die, die du zusammen mit Berenike benutzt hast. Sie möchte nun den gesamten Parkettboden mit den beiden Rauten auslegen. Dafür muss sie wissen wie sie weiter legen kann, damit sich keine Überlappungen ergeben. Nicht mit allen Anordnungen, die du beim Lösen dieser „Mathe im Advent"-Aufgabe gefunden hast, klappt das.

Diese sogenannten aperiodischen Parkettierungen findet man nicht nur in der Mathematik. Sie lassen sich auch bei der Untersuchung einiger Materialien wiederfinden. Dabei sind die Atome (kleinsten Bestandteile) des Materials wie in Penrose-Kacheln angeordnet.

Übrigens, den Nobelpreis hat Roger Penrose nicht für die Entdeckung des Penrose-Parketts bekommen. Er hat nachgewiesen, dass Schwarze Löcher wirklich existieren. Wie auf der Webseite der Deutschen MathematikerVereinigung www.mathematik.de zu lesen ist, hat Penrose bewiesen, dass Schwarze Löcher eine direkte Folge der allgemeinen Relativitätstheorie von Albert Einstein sind. Im Jahr 1965, zehn Jahre nach Einsteins Tod, bewies Penrose, dass Schwarze Löcher wirklich entstehen können, und beschrieb sie im Detail. Ein schwarzes Loch hat keine Oberfläche wie ein Planet oder ein Stern. Es ist hingegen ein Gebiet im Weltraum, in dem die Materie in sich selbst zusammengefallen ist. Dieser Kollaps von katastrophalen Ausmaßen bewirkt, dass sich eine enorme Menge Masse auf einem unglaublich winzigen Raum konzentriert. Mathematisch gesehen sind es Singularitäten, in denen alle uns bekannten Naturgesetze in sich zusammenbrechen, schrieb die Deutsche Physikalische Gesellschaft am 6. Oktober 2020, dem Tag der Bekanntgabe der Nobelpreise, in ihrer Pressemitteilung. 